\title{
Sensitivity to the context of facial expression in the still face at 3-, 6-, and 9-months of age
}

\author{
Tricia Striano $^{\mathrm{a}, \mathrm{b}, \mathrm{c}, *}$, Ulf Liszkowski ${ }^{\mathrm{a}}$ \\ Cultural Ontogeny Group \\ ${ }^{a}$ Max Planck Institute for Evolutionary Anthropology, Cultural Ontogeny, Deutscher Platz 6, \\ Leipzig 04103, Germany \\ ${ }^{\mathrm{b}}$ Max Planck Institute for Human Cognitive and Brain Sciences, Stephanstrasse 1a, Leipzig, Germany \\ ${ }^{\mathrm{c}}$ University Leipzig, Center for Advanced Studies, Leipzig, Germany
}

Received 13 April 2004; received in revised form 21 June 2004; accepted 22 June 2004

\begin{abstract}
Thirty-eight 3-, 6-, and 9-month-old infants interacted in a face to face situation with a female stranger who disrupted the on-going interaction with $30 \mathrm{~s}$ Happy and Neutral still face episodes. Three- and 6-month-olds manifested a robust still face response for gazing and smiling. For smiling, 9-month-olds manifested a floor effect such that no still face effect could be shown. For gazing, 9-month-olds' still face response was modulated by the context of interaction such that it was less pronounced if a happy still face was presented first. The findings point to a developmental transition by the end of the first year, whereby infants' still face response becomes increasingly influenced by the context of social interaction.
\end{abstract}

(C) 2004 Published by Elsevier Inc.

Keywords: Facial expression; Still face; Interaction

\section{Introduction}

The still face paradigm has been widely used to assess human infants' early social expectations (Adamson \& Frick, 2003; Tronick, Als, Adamson, Wise, \& Brazelton, 1978). As young as 2-months

\footnotetext{
* Corresponding author. Tel.: +49 3419940 123; fax: +49 3413550119.

E-mail address: striano@ cbs.mpg.de (T. Striano).
} 
of age, infants avert their gaze, smile less, and self-comfort when they encounter a suddenly still faced partner who had been communicating with them (Cohn \& Tronick, 1987; Field, Vega-Lahr, Goldstein, \& Scafidi, 1986; Toda \& Fogel, 1993). Such findings index infants' rudimentary expectations about the nature of face to face interactions with people (see also Gusella, Muir, \& Tronick, 1988; Muir \& Hains, 1993; Rochat \& Striano, 1999).

Modifications of the still face paradigm reveal that the still face response is robust but also selective. Based on a variety of careful manipulations to rule out alternative explanations, it has been shown that the still face situation is not due simply to a general loss of stimulation or fatigue over time (e.g., Gusella et al., 1988; see also Muir \& Hains, 1993). The still face effect is reserved for people and not objects (i.e., Ellsworth, Muir, \& Hains, 1993), and shows that infants are attuned to more than the presence or absence of stimulation.

While the still face paradigm has been systematically used with infants in the first half of the first year, it has not been commonly used to investigate the social competencies of older infants. Once infants start to systematically coordinate attention between people and objects by 9-12 months of age (i.e., Tomasello, 1999), researchers have turned to triadic paradigms which involve the integration of people and objects (e.g., Bakeman \& Adamson, 1984; Carpenter, Nagell, \& Tomasello, 1998). However, the still face effect may be used to track the development of social competencies over the course of the first year (see also Striano \& Rochat, 1999).

The current study assessed infants' still face responses at 3, 6, and 9 months of age. The procedure was based on prior research that assessed infants' response to "emotional" still faces. In a study with 5-month-old infants, D'Entremont and Muir (1997) found that infants manifested a small but significant effect for smiling when their mother presented a happy still face compared to a neutral and sad still face. Taking a developmental approach, Rochat, Striano, and Blatt (2002) compared 2-, 4-, and 6-month-olds' responses to $30 \mathrm{~s}$ Happy, Neutral, and Sad still face episodes presented by a stranger. Two-month-olds did not show a reduction in gazing for the Happy still face. The authors suggest that between 4 and 6 months of age, infants become more sensitive to the timing and structure of face to face interactions (see also Rochat, Querido, \& Striano, 1999), whereas younger infants are focused on the presence or absence of positive facial expressions.

In the current study, we extended the research of D'Entremont and Muir (1997) and Rochat et al. (2002) by presenting different emotional still faces to 3-, 6-, and 9-month-old infants. Developmental transitions that happen by the end of the first year, such as the capacity for joint attention (Carpenter et al., 1998) and social re-engagement efforts (Cohn \& Tronick, 1987; Striano \& Rochat, 1999) are thought to reflect, in part, the infants' developing capacity to understand the underlying motives and goals of others. Considering prior actions assists in understanding the meaning of others' behaviors (i.e., Carpenter, Call, \& Tomasello, 2002). Similarly, a sensitivity to others' facial expressions is a necessary precursor to recognizing the meaning behind others' emotions and predicting how people will behave in the future (Walker-Andrews, 1997). Interestingly, by the end of the first year, infants categorize various static expressions based on their underlying meaning rather than perceptual similarity (i.e., Ludemann, 1991; Nelson, 1987), and to use these expressions to interpret others' future actions (Phillips, Wellman, \& Spelke, 2002; Poulin-Dubois, 1999) and to guide their own behavior (Campos \& Sternberg, 1981; Moses, Baldwin, Rosicky, \& Tidball, 2001; Sorce, Emde, Campos, \& Klinnert, 1985).

In the current study, infants were presented between normal interaction periods with a happy still face and a neutral still face episode, with the order counterbalanced. We expected that infants at all ages would discriminate facial expressions (see Nelson, 1987; Serrano, Iglesias, \& Loeches, 1992; Striano, 
Brennan, \& Vanman, 2002; Walker-Andrews, 1997), but that the context of these expressions which were presented after a normal interaction period and during a sudden still-face period would modulate infants' behavioral response. In particular, we expected that by 3 months of age infants would show sensitivity to the static facial expression posed during the still face episode. In particular, following Rochat et al.'s interpretation that young infants before 4 months of age show selective attunement to positive affect mirroring and expressions, we expected that this age group would show a reduced still face effect when a happy expression was posed. We predicted that they would not be influenced by the order of the still face expressions. Following prior research (i.e., Rochat et al., 2002; see also Rochat \& Striano, 1999) we expected that infants at 6 months of age would not respond differentially to the emotional still faces. At this age, research suggests that infants are primarily attuned to the presence or absence of interpersonal contact, and not to the underlying reason behind a loss of social contingencies (Delgado, Messinger, \& Yale, 2002), or emotional expressions posed during the break of contact.

We expected a different pattern for the 9-month-olds, such that they would be influenced by the emotion posed during the still face episode, and also by the order of the emotion presentation. By the end of the first year, categorize emotional expressions based on their meaning rather than perceptual characteristics (i.e., Ludemann, 1991; however see also Bornstein \& Arterberry, 2003), and begin to interpret others' behavior as intentional (Tomasello, 1995). Thus, we expected that the context of the still face expression would influence infants' responses at this age. We expected a reduced still face effect when the happy expression was presented first, given that it fell in the context of a preceding positive and reciprocal interaction. When the happy expression was presented second (i.e., following experience with a Neutral still face episode), we expected 9-month-olds to show a more robust still face effect given the prior ambiguous emotional context.

\section{Method}

\subsection{Participants}

Forty-eight infants were tested. Ten infants were excluded because of fussiness (seven), or because they refused to sit in the chair (three). A final sample of 38 infants was included: 133 -month-olds (mean $=102$ days; range = 93-109 days), 13 6-month-olds (mean = 199 days; range = 187-210 days), and 12 9-montholds ( mean $=295$ days; range $=281-305$ days). Parents were contacted by phone and invited to participate with their infants. All infants were White and from lower to middle-class backgrounds, and living in the east of Germany. Infants were given a small gift for participating in the study.

\subsection{Procedure}

Infants were videotaped while interacting with a female adult stranger sitting across from them $2.5 \mathrm{ft}$ away with their faces aligned for eye contact. Three and 6-month-old infants were placed in a commercial upright infant seat resting on a table, 9-month-olds were seated in a high-chair.

Infants and experimenter faced each other in four alternating 30-s episodes totaling $120 \mathrm{~s}$. The testing always began with a 30-s episode of normal interaction. Each 30-s episode of normal interaction was followed by a 30-s episode of still face. 
Each still face episode varied according to the emotional expression adopted by the experimenter while displaying a frozen face, staring toward the infant's eyes whenever the infant was looking toward her. The experimenter was blind to the experimental hypotheses. In the Neutral still face, the experimenter held a neutral, resting expression with her mouth closed and relaxed. In the Happy still face, the experimenter assumed a large, toothy, static smile with an upturned mouth and lifted cheeks. During the normal interaction episodes, the experimenter actively engaged the infants and maintained eye contact, she talked to them and displayed dynamic facial expressions, but never touched them. The order of still face emotions (Happy, Neutral) was counterbalanced across infants of each age group. Parent(s) watched their infant on a TV monitor which was hidden behind a curtain. The experimenter (E1) was cued by an assistant standing behind the infant who timed the experiment and signaled when to start and end a particular episode.

\subsection{Apparatus}

Experimenter and infant were videotaped via four synchronized cameras (Panasonic NV-DS 150). The four images were mixed and recorded via a video splitter and VCR (Panasonic Quad System WJ-MS 424). Cameras were placed on tripods providing frontal and side views of the experimenter's and the infant's face.

\subsection{Coding and reliability}

Gazing and smiling were coded and measured in percent of the total duration of an episode during the testing session, and operationally defined as:

Gazing: looking toward the experimenter's face.

Smiling: upturned motion of at least one corner of the mouth, raising of the cheeks with gazing to E. Because we were interested in infants' social response directed at E, only smiling that was accompanied by gazing was coded.

Coding of smiling and gazing was performed off-line and in real-time with a specialized program (Interact, Version 6.5, Thiel, 1991) using a frame-by-frame coding function ( 25 frames/s) with on/off activation of a keyboard key corresponding to the occurrence of either gazing or smiling. Interact calculated the percent of the total episode time the key was activated. When necessary, the coding could be done in slow motion.

For reliability, two independent coders blind to the hypothesis analyzed $20 \%$ of the infants of all ages. Cohen-Kappas for both gazing and smiling on a 1-s time-base were 0.96 and 0.84 , respectively.

\section{Results}

Analyses were carried out separately for gazing and smiling. Following D'Entremont and Muir (1997), we performed a four (episode: Normal 1, Still face 1, Normal 2, Still face 2) $\times$ two (order: Happy still face first, Neutral still face first) $\times$ three (age: 3, 6, and 9 months) ANOVA. To test a main effect of episode we performed two planned contrasts. First, we compared the two normal episodes to the two still face episodes (the still face effect). Second, to check for behavioral changes over time we compared the 
first normal episode to the second normal episode. To investigate the effect of the different emotional expressions, we conducted a two (emotion: Happy still face, Neutral still face) $\times 2$ (order: Happy still face first, Neutral still face first) $\times 3$ (age: 3-, 6-, 9-months) ANOVA. Preliminary analyses with a four (episode) $\times 2($ sex $)$ ANOVA on effects of gender did not yield significant differences, so this variable was collapsed for further analyses.

Table 1 provides the means and standard errors of means of each episode and age group for gazing and smiling. As indicated in Table 1, there was an overall episode effect for gazing and smiling response (respectively, $F(3,96)=31.6 ; p<0.001 ; F(3,96)=16.2 ; p<0.001)$. Planned contrasts confirmed a still face effect with a reduction in gazing and smiling during the still face episodes relative to the normal interactions (respectively, $F(1,32)=65.6, p<0.001 ; F(1,32)=35.8, p<0.001$ ), and showed that there were no significant difference between the normal episodes ( $p$ 's $>0.1)$, thus ruling out fatigue. There were age effects, such that 3-month-olds gazed and smiled significantly more than 6- and 9-month-olds (Tukey post hoc, $p$ 's $<0.02$ ).

For gazing there was an age $\times$ episode interaction $(F(6,96)=2.3, p=0.038)$. Analyzing each age group separately confirmed a significant still face effect in each age group ( $p$ 's $<0.01)$. As indicated in Table 1, the interaction likely rests on a more pronounced still face effect for the group of 3-month-olds.

There was also an age $\times$ episode effect for smiling $(F(6,96)=4.6, p<0.001)$. The interaction was due to a significant reduction in smiling during the still face for both 3- and 6-month-old infants, and no reduction for 9-month-old infants. However, it is important to note that less than half of the 9-month-olds smiled at $\mathrm{E}$ in any of the four episodes (number of smiling infants per episode: Normal $1=5$; $\mathrm{SF} 1=4$; Normal 2=3; SF2 = 1) such that no still face effect could be confirmed for this age group.

Comparison of the emotional expressions for gazing revealed a significant order $\times$ age interaction $(F$ $(2,32)=3.7, p=0.036)$. Fig. 1 shows infants' gazing as a function of both orders. As shown by comparing the right and left panels of Fig. 1, 9-month-olds gazed reliably more in the Happy still face episode when the Happy still face was presented first $(F(1,10)=11.50, p=0.007)$.

For smiling, there was a significant order $\times$ emotion interaction $(F(2,32)=4.1, p=0.049)$ suggesting that infants smiled significantly more to the Happy than Neutral still face in the Happy Order $(F(1$, $17)=9.4, p=0.008)$. However, given the floor effect in the 9-month-olds and a marginal age effect $(F(2$,

Table 1

Mean statistics for gazing and smiling by age and episode

\begin{tabular}{|c|c|c|c|c|c|}
\hline Age group & Measure & Normal interaction $1^{\mathrm{a}}$ & Still face $1^{\mathrm{a}}$ & Normal interaction $2^{\mathrm{a}}$ & Still face $2^{\mathrm{a}}$ \\
\hline \multirow[t]{2}{*}{ 3-month-olds ${ }^{\mathrm{b}}$} & Gazing & $92.66(4.80)^{\mathrm{c}}$ & $56.78(8.89)^{\mathrm{d}}$ & $94.33(2.48)^{\mathrm{c}}$ & $53.55(7.82)^{\mathrm{d}}$ \\
\hline & Smiling & $33.29(6.7)^{\mathrm{c}}$ & $9.46(2.64)^{\mathrm{d}}$ & $27.19(6.98)^{\mathrm{c}}$ & $4.64(2.13)^{\mathrm{d}}$ \\
\hline \multirow[t]{2}{*}{ 6-month-olds } & Gazing & $53.86(7.42)^{\mathrm{c}}$ & $33.77(9.05)^{\mathrm{d}}$ & $44.63(7.52)^{\mathrm{c}}$ & $31.35(6.77)^{\mathrm{d}}$ \\
\hline & Smiling & $17.89(5.26)^{\mathrm{c}}$ & $2.38(1.33)^{\mathrm{d}}$ & $8.32(4.80)^{\mathrm{c}}$ & $2.11(1.16)^{\mathrm{d}}$ \\
\hline \multirow[t]{2}{*}{ 9-month-olds } & Gazing & $53.13(7.39)^{\mathrm{c}}$ & $34.19(5.79)^{\mathrm{d}}$ & $59.20(5.32)^{\mathrm{c}}$ & $26.96(6.11)^{\mathrm{d}}$ \\
\hline & Smiling $^{\mathrm{e}}$ & $3.83(1.54)$ & $3.16(1.58)$ & $3.53(1.54)$ & $1.58(1.58)$ \\
\hline
\end{tabular}

Superscripts indicate significant differences $(p<0.05)$ according to the analyses reported.

a Significant main effect.

b Significantly different from 6- to 9-month-olds.

c Not significantly different.

d Significantly different from normal interactions.

e No statistics are computed because less than half of the infants smiled. 
$\square$ Normal Interaction 目Happy Still Face $\square$ Normal Interaction $\square$ Neutral Still Face

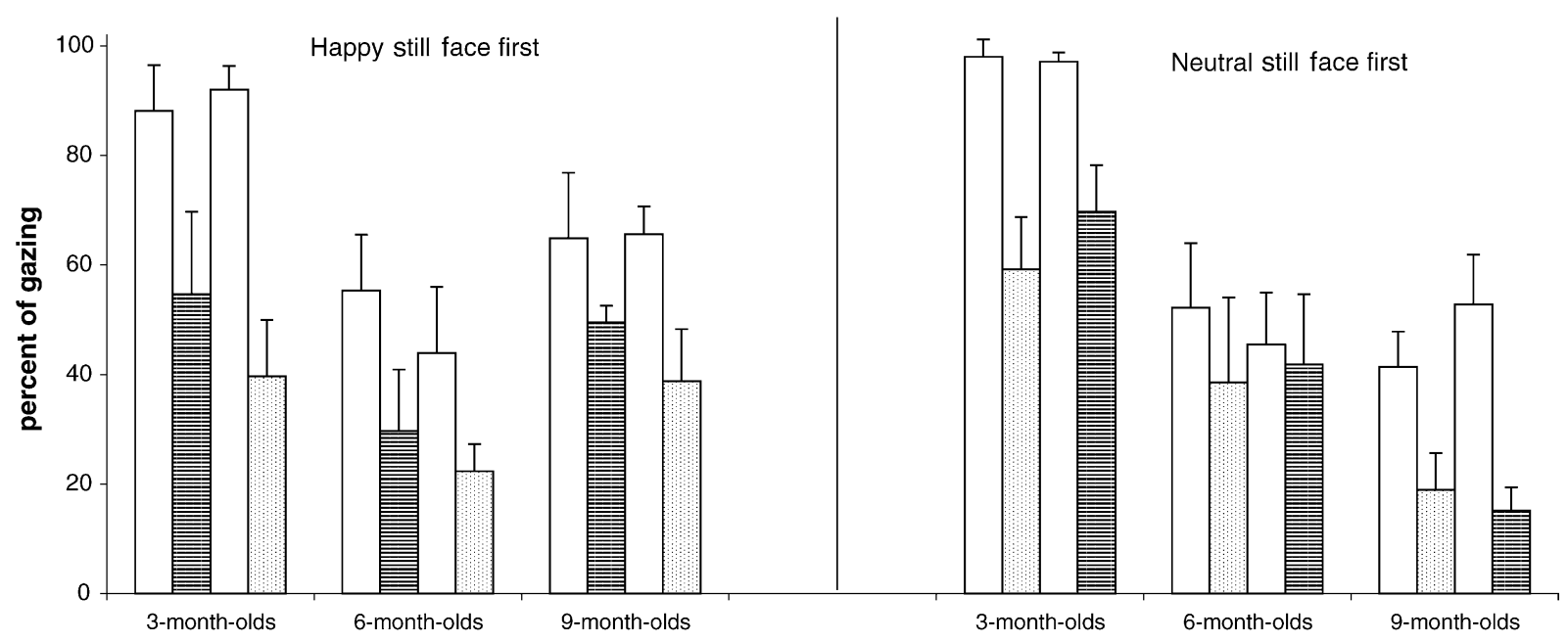

Fig. 1. Normal and Still face episodes for gazing. Left and right panel present, respectively, the order with the Happy or Neutral still face first.

$32)=3, p=0.064)$, the order effect for smiling was analyzed for each age group separately. Fig. 2 shows that 3- but not 6-month-olds displayed an order $\times$ emotion interaction $(F(1,11)=6.13, p=0.031)$. At 3 months, infants smiled significantly more to the first still face regardless of the order of expression.

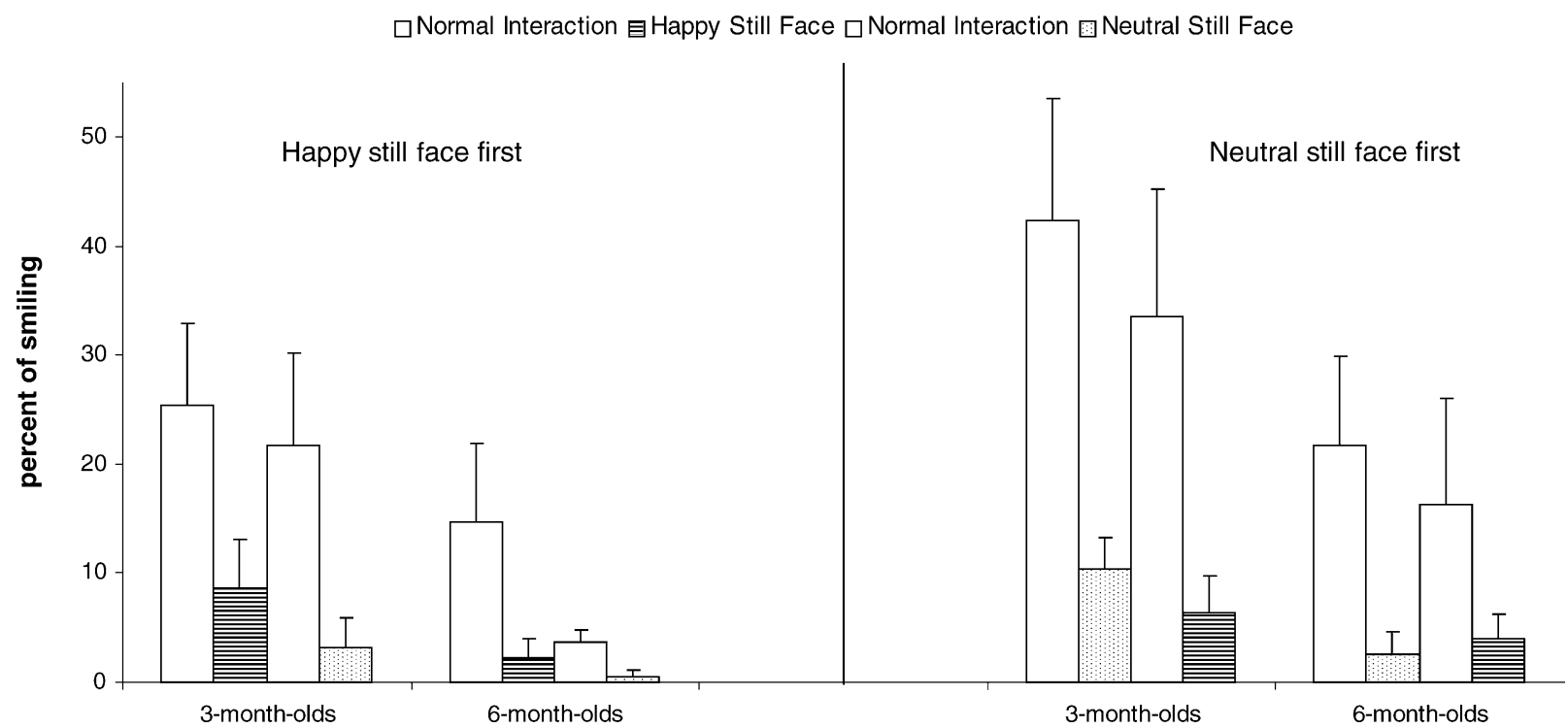

Fig. 2. Normal and Still face episodes for smiling. Left and right panel present, respectively, the order with the Happy or Neutral still face first. 


\subsection{Summary of main results}

Three- and 6-month-olds showed a significant still face effect for both expressions for gazing and smiling. Three-month-olds smiled more at the experimenter in the first still face episode, regardless of which expression was presented. For smiling, 9-month-olds showed a floor effect. For gazing, there was an order by age interaction such that 9-month-olds gazed reliably more to the still faces when the Happy expression was presented first.

\section{Discussion}

In the current study, 3-, 6-, and 9-month-olds' response to emotional still faces was assessed. In all, we found that 3-and 6-month-olds manifested a still face response for both emotional expressions, gazing and smiling at the experimenter reliably more in the normal interaction phase compared to the still face episode. However, 9-month-olds' gazing was modulated by the context of preceding interaction episodes, and their frequency of smiling was at floor for all episodes.

Infants' behavior toward the experimenter was modulated by age as well as the order in which the expressions were presented. We predicted that at 3 months of age, infants would show a reduced still face response to the Happy still face episode. This prediction was not confirmed, and did not therefore replicate Rochat et al.'s finding with 2-month-old infants. We found that infants at 3 months of age manifested a still face response regardless of the expression posed. They smiled less to the second still face, regardless of emotion, indicating an overall reduction of social responsiveness in a second still face episode. In general, the effect for infants at 3 months of age are similar to those reported by D'Entremont and Muir with 5-month-olds; whereby the greatest effects were due to changes in contingency (i.e., normal versus still face, and not to the emotion posed during the still face episode).

Rochat et al. suggest a transition between 2 and 4 months in terms of the determinants of infants' sensitivity to social contingencies (see also Rochat et al., 1999). The current study points to a potential developmental transition between 2 and 3 months, whereby infants might start to pay more attention to the contingency of expressions (i.e., how these expressions relate to on-going interaction) rather than to the mere presence or absence of expressions. This transition has been suggested by other researchers (e.g., Bigelow, 2001; D’Entremont \& Muir, 1997; Nadel, Kervella, Marcelli, \& Reserbat-Plantey, 1999; Rochat \& Striano, 1999) and deserves further attention.

In relation to effects of the context of interaction, gazing responses revealed interesting developmental differences which interacted with the order of stimulus presentation. In particular, 9-month-olds gazed reliably more in both still face episodes when the first still face had a Happy expression. This finding likely reflects infants' developing awareness of others, whereby infants become sensitive to previous interactions when reciprocating with and making predictions about others.

Whereas the trend for 3- and 6-month-olds was an increase in gazing in the Happy still face condition regardless of order, 9-month-olds may have been turned off by the experimenter when the neutral expression was presented first. The results for 9-month-olds, especially in relation to smiling, might also reflect changes in attachment and stranger anxiety also occurring around this age (e.g., Bretherton, 2003). Future studies should assess in more detail whether the developmental differences reported here reflect a sensitivity to the context of emotions or index an artifact of stranger anxiety or other cognitive developments. Comparing infants' response to the context of expressions posed by 
familiar and unfamiliar adults would be one way to assess the generalizability and meaning of these findings.

In general, the study points to a developmental transition in the interpretation of facial expressions that is dependent upon the context of interaction. This is the first study to assess infants' understanding of the context of expressions developmentally and up to 9 months of age. Future studies should assess further these relations by varying the context of emotions - it would be revealing, for instance, to present infants of different ages with repeated positive still face episodes (i.e., Happy SF, Happy SF) and compare their responses to more ambiguous negative-positive episodes (i.e., Negative SF, Happy SF). In addition, it would be useful to compare the influence of the context of other expressions such as fear, surprise, or disgust on infants' responsiveness.

We argue that a consideration of the context in which expressions occur (see also Montague \& WalkerAndrews, 2001), whether following or preceding positive or neutral expressions, is a necessary step to understanding the underlying meaning of expressions. In sum, the current study confirms the use of the still face paradigm in infants across the first year. The findings show that by 3-4 months of age infants are sensitive to contingency regardless of emotional expressions accompanying it, and point to developmental transitions by the end of the first year when context of interactions is taken into account. The key is to establish the mechanisms that promote such developments and to establish how such skills may relate to the human ability to interpret others' behavior and expressions.

\section{Acknowledgements}

We are grateful to Saskia Stieber and Julian Heister for assistance with running the study and to the Universitatsfrauenklinik for support and assistance with subject recruitment. We are grateful to Daniel Stahl for statistical comments. We thank the parents and infants for participating in the research project.

\section{References}

Adamson, L. B., \& Frick, J. E. (2003). The still face: A history of a shared experimental paradigm. Infancy, 4, 451-473.

Bakeman, R., \& Adamson, L. B. (1984). Coordinating attention to people and objects in mother-infant and peer-infant interaction. Child Development, 55, 1278-1289.

Bigelow, A. E. (2001). Discovering self through other: Infants' preference for social contingency. Bulletin of the Menninger Clinic, 65, 335-346.

Bretherton, I. (2003). Continued reflections on the internal working model construct and its relevance to attachment relationships. Talk presented at: Attachment from Infancy and Childhood to Adulthood. Germany: Regensburg; 11-13 July.

Bornstein, M., \& Arterberry, M. (2003). Recognition, discrimination, and categorization of smiling by 5 month old infants. Developmental Science, 6, 585-599.

Campos, J. J., \& Sternberg, C. R. (1981). Perception, appraisal, and emotion: The onset of social referencing. In M. E. Lamb \& L. R. Sherrod (Eds.), Infant social cognition: Empirical and theoretical considerations (pp. 273-314). Hillsdale: Lawrence Erlbaum Association.

Carpenter, M., Call, J., \& Tomasello, M. (2002). Understanding "prior intentions" enables two-year-olds to imitatively learn a complex task. Child Development, 73, 1431-1441. 
Carpenter, M., Nagell, K., \& Tomasello, M. (1998). Social cognition, joint attention, and communicative competence from 9 to 15 months of age. Monographs of the Society of Research in Child Development, 63, Serial No. 176.

Cohn, J. F., \& Tronick, E. (1987). Mother-infant face-to-face interaction: The sequence of dyadic states at 3, 6 and 9 months. Developmental Psychology, 23, 68-77.

Delgado, C., Messinger, D., \& Yale, M. (2002). Infant response to direction of parental gaze: A comparison of two still-face conditions. Infant Behavior and Development, 25, 311-318.

D'Entremont, B., \& Muir, D. W. (1997). Five-month-olds' attention and affective responses to still-faced emotional expressions. Infant Behavior and Development, 20, 563-568.

Ellsworth, C. P., Muir, D., \& Hains, S. M. (1993). Social competence and person-object differentiation: An analysis of the still-face effect. Developmental Psychology, 29, 3-73.

Field, T., Vega-Lahr, N., Goldstein, S., \& Scafidi, F. (1986). Face-to-face interaction behavior across early infancy. Infant Behavior and Development, 10, 111-116.

Gusella, J. L., Muir, D., \& Tronick, E. A. (1988). The effect of manipulating maternal behavior during an interaction on 3- and 6-month-olds' affect and attention. Child Development, 59, 1111-1124.

Ludemann, P. M. (1991). Generalized discrimination of positive facial expressions by 7- and 10-month-old infants. Child Development, 62, 55-67.

Montague, D. P. F., \& Walker-Andrews, A. S. (2001). Peekaboo: A new look in infants' perception of emotion expressions. Developmental Psychology, 73, 1339-1352.

Moses, L. J., Baldwin, D. A., Rosicky, J. G., \& Tidball, G. (2001). Evidence for referential understanding in the emotions domain at 12 and 18 months. Child Development, 72, 718-735.

Muir, D. W., \& Hains, S. M. (1993). Infant sensitivity to perturbations in adult facial, vocal, tactile and contingent stimulation during face-to-face interactions. In B. de Boysson-Bardies \& S. de Schonen, et al. (Eds.), Developmental neurocognition: Speech and face processing in the first year of life: vol. 69 (pp. 171-185). Amsterdam: Elsevier.

Nadel, J., Carchon, I., Kervella, C., Marcelli, D., \& Reserbat-Plantey, D. (1999). Expectancies for social contingency in 2-montholds. Developmental Science, 2, 164-173.

Nelson, C. A. (1987). The recognition of facial expressions in the first two years of life: Mechanisms of development. Child Development, 58, 889-909.

Phillips, A. T., Wellman, H. M., \& Spelke, E. S. (2002). Infants' ability to connect gaze and emotional expressions to intentional actions. Cognition, 85, 53-78.

Poulin-Dubois, D. (1999). Infants' distinction between animate and inanimate objects: The origins of naive psychologie. In P. Rochat (Ed.), Early social cognition: Understanding others in the first months of live (pp. 257-281). Hillsdale: Lawrence Erlbaum Associates.

Rochat, P., Querido, J., \& Striano, T. (1999). Emerging sensitivity to the timing and structure of protoconversation in early infancy. Developmental Psychology, 35, 950-957.

Rochat, P., \& Striano, T. (1999). Social-cognitive development in the first year. In P. Rochat (Ed.), Early social cognition: Understanding others in the first months of life. Mahwah: Lawrence Erlbaum Association.

Rochat, P., Striano, T., \& Blatt, L. (2002). Differential effects of happy, neutral, and sad still face on 2-, 4- and 6-month-old infants. Infant and Child Development, 11, 289-303.

Serrano, J. M., Iglesias, J., \& Loeches, A. (1992). Visual discrimination and recognition of facial expressions of anger, fear, and surprise in 4-6-month-old infants. Developmental Psychobiology, 25, 411-425.

Sorce, J. F., Emde, R. N., Campos, J., \& Klinnert, M. D. (1985). Maternal emotional signaling: Its effect on the visual cliff behavior of 1-year-olds. Developmental Psychology, 21, 195-200.

Striano, T., \& Rochat, P. (1999). Developmental link between dyadic and triadic social competence in infancy. British Journal of Developmental Psychology, 17, 551-562.

Striano, T., Brennan, P., \& Vanman, E. (2002). Maternal Depressive Symptoms and 6-month-old infants' sensitivity to facial expressions. Infancy, 3, 115-126.

Thiel, T. (1991). Interact (Version 6.5). Germany: Berlin.

Toda, S., \& Fogel, A. (1993). Infant response to the still-face situation at 3 and 6 months. Developmental Psychology, 29, 532-538.

Tomasello, M. (1995). Joint attention as social cognition. In C. Moore \& P. Dunham (Eds.), Joint attention: Its origins and role in development. Hillsdale: Lawrence Erlbaum Associates. 
Tomasello, M. (1999). The cultural origins of human cognition. Cambridge, MA: Harvard University Press.

Tronick, E. Z., Als, H., Adamson, L., Wise, S., \& Brazelton, T. B. (1978). The infant's response to entrapment between contradictory message in face-to-face interaction. Journal of the American Academy of Child Psychiatry, 16, 1-13.

Walker-Andrews, A. S. (1997). Infants' perception of expressive behaviors: Differentiation of multimodal information. Psychological Bulletin, 121, 437-456. 